\title{
Canakinumab with and without pembrolizumab in patients with resectable non-small-cell lung cancer: CANOPY-N study design
}

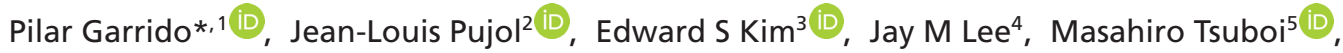
Ana Gómez-Rueda1', Amparo Benito", Nicolas Moreno', Luis Gorospe1, Tuochuan Dong ${ }^{6}$, Cecile Blin 7 , Vanessa Rodrik-Outmezguine ${ }^{6}$ (D), Vanessa Q Passos ${ }^{6}$ \& Tony SK Mok ${ }^{8}$ (D)

${ }^{1}$ Hospital Ramón y Cajal, Madrid, 28034, Spain

${ }^{2}$ Hospital Arnaud de Villeneuve, Montpellier, 34090, France

${ }^{3}$ Levine Cancer Institute, Atrium Health, Charlotte, NC 28202, USA

${ }^{4}$ David Geffen School of Medicine at UCLA, Los Angeles, CA 90095, USA

${ }^{5}$ National Cancer Center Hospital East, Kashiwa, 112-0002, Japan

${ }^{6}$ Novartis Pharmaceuticals Corporation, East Hanover, NJ 07936, USA

${ }^{7}$ Novartis Pharma AG, Basel, CH-4056, Switzerland

${ }^{8}$ The Chinese University of Hong Kong, Hong Kong, 999077, China

*Author for correspondence: Tel.: +34 913368 263; pilargarridol@gmail.com

Canakinumab is a human IgGk monoclonal antibody, with high affinity and specificity for IL-1 $\beta$. The Canakinumab Anti-Inflammatory Thrombosis Outcome Study (CANTOS) trial, evaluating canakinumab for cardiovascular disease, provided the first signal of the potential of IL-1 $\beta$ inhibition on lung cancer incidence reduction. Here, we describe the rationale and design for CANOPY-N, a randomized Phase II trial evaluating IL-1 $\beta$ inhibition with or without immune checkpoint inhibition as neoadjuvant treatment in patients with non-small-cell lung cancer. Patients with stage IB to IIIA non-small-cell lung cancer eligible for complete resection will receive canakinumab or pembrolizumab as monotherapy, or in combination. The primary end point is major pathological response by central review; secondary end points include overall response rate, major pathological response (local review), surgical feasibility rate and pharmacokinetics.

Clinical trial registration: NCT03968419 (ClinicalTrials.gov)

Lay abstract: A previous study showed that canakinumab reduced the risk of lung cancer in patients with heart disease. Canakinumab blocks an inflammatory protein called IL-1 $\beta$ that is involved in cancer. Anticancer drugs used before surgery ('neo-adjuvant') can improve the success rate of surgery and may help prevent the cancer from returning. Neo-adjuvant trials help us understand how the drugs work and how they affect cancer. CANOPY-N (NCT03968419) is an ongoing randomized, exploratory, Phase II clinical trial testing canakinumab and pembrolizumab (a different cancer immunotherapy), alone or combined, for patients with early non-small-cell lung cancer. The study will test whether treatment can kill most cancer cells in the surgery sample ('major pathological response'). It will also investigate other effects on cancer biology, levels of molecules that measure possible clinical benefit ('biomarkers') and side effects.

First draft submitted: 28 October 2020; Accepted for publication: 9 February 2021; Published online: 2 March 2021

Keywords: canakinumab • CANOPY • early stage • immunotherapy • major pathological response • MPR - neoadjuvant • non-small-cell lung cancer • resection • surgery

In 2018, there were approximately 2,094,000 new cases of lung cancer worldwide, and its incidence is estimated to increase by $38 \%$ by 2030 [1]. Lung cancer is the most commonly diagnosed cancer $(11.6 \%)$ and the leading cause of cancer deaths (18.4\%) [2]. Non-small-cell lung cancer (NSCLC) accounts for $85 \%$ of lung cancer diagnoses [3]. 
Accurate staging of the disease determines the prognosis and treatment approach. Most patients present with metastatic disease at diagnosis, for which 5-year survival is still very poor [4]. Up to $22 \%$ of patients present at diagnosis with early-stage lung cancer (stages I, II and selected stage IIIA NSCLC with a possibility of complete tumor resection) [5]. For this group of patients, surgical resection with curative intent is the standard-of-care [6]. However, recurrence rates are high, ranging between 20 and 50\% [6-8]. The 5-year survival of early-stage NSCLC is approximately $50 \%$, with most patients dying of their underlying disease [6].

The high incidence of distant recurrence suggests that systemic therapies are required to improve cure rates. The benefits of neoadjuvant chemotherapy have been assessed in many trials. A meta-analysis of 15 randomized controlled trials showed a significant benefit of preoperative chemotherapy on survival, with a $13 \%$ reduction in the relative risk of death. This finding represents an absolute survival improvement of $5 \%$ at 5 years (from 40 to 45\%) [9]. A meta-analysis of eight trials including 1586 patients showed that patients with resectable stage IIIA NSCLC treated with neoadjuvant chemotherapy followed by surgery had a $16 \%$ reduction in their risk of death compared with surgery alone [10]. These modest improvements in survival with neoadjuvant chemotherapy underpin the need to evaluate treatment modalities with a different mechanism of action and/or combination therapies to increase curability in early-stage lung cancer.

The main objective of neoadjuvant treatment is to reduce the size of the primary tumor, thus increasing the likelihood of complete surgical resection, and to control micro-metastases, the primary cause of relapse, as early as possible [11]. Due to the availability of resected tumor tissue from patients without any prior treatment, the neoadjuvant setting provides the unique opportunity to assess the impact of treatment on the tumor microenvironment (TME); it is also an opportunity to determine which signaling pathways are involved in resistance to therapy and to inform further treatment plans. Additionally, there is an opportunity to explore potential predictive biomarkers of response to treatment.

Monoclonal antibodies that target the PD-1 receptor or the PD-L1 have demonstrated improved efficacy and favorable side effect profiles compared with chemotherapy in first-line treatment in patients with metastatic NSCLC [12,13] and as consolidation therapy after definitive chemoradiation in unresectable stage III disease [14]. These findings raised the possibility of enhancing the curability of earlier disease stages using these systemic therapies. Several PD-1/PD-L1 inhibitors (e.g., pembrolizumab, nivolumab, atezolizumab and durvalumab) have been approved in various lines of therapy, which prompted investigation of their use in earlier stages of the disease when the tumor is potentially 'resectable for cure' $[11,15,16]$. Based on the hypothetical advantage of an intact locoregional immune environment including the primary tumor and lymph node drainage system, the neoadjuvant setting could represent the optimal line of treatment for immunotherapy [15]. In situ tumors may provide a more substantial neoantigen load compared with the adjuvant setting, hence allowing enhanced immune recognition and drug efficacy [17].

In clinical research trials, the neoadjuvant setting is useful for evaluation of the biologic and clinical effects of treatment and can accelerate discovery of new curative therapies [6]. Notably, tissue samples are readily available both before and after treatment in this setting, which facilitates investigation of tissue-based biomarkers. Major pathological response (MPR), defined as $\leq 10 \%$ residual viable tumor cells in the surgical resection specimen, has been recognized as a potential surrogate end point in neoadjuvant clinical trials in patients with NSCLC [18]. MPR has shown a positive correlation with both disease-free survival (DFS) and overall survival (OS) [19].

Recent clinical studies have published results of first analyses of the safety and feasibility of the use of neoadjuvant anti-PD-1/PD-L1 inhibitors in some patients with NSCLC (Table 1) [20-27]. In a pilot Phase II study of 21 eligible patients with surgically resectable NSCLC (stage I, II or IIIA) conducted by Forde et al., nivolumab monotherapy administered as neoadjuvant treatment $(3 \mathrm{mg} / \mathrm{kg}$ for two cycles during 4 weeks before surgery) did not delay surgery and induced MPR in 45\% of resected tumors (9/20). Nivolumab-related side effects of any grade occurred in $23 \%(5 / 22)$ of patients [20]. In a different Phase II trial investigating toxicity, efficacy and surgical outcomes of neoadjuvant pembrolizumab (200 mg for two cycles prior to surgery) in 30 patients with resectable NSCLC, at least two pathological complete responses (pCRs) were observed [21]. In a single-arm Phase II trial, Shu et al. reported that $57 \%(17 / 30)$ and $33 \%(10 / 30)$ of patients with stage IB-IIIA NSCLC treated with two cycles of neoadjuvant atezolizumab $\left(1200 \mathrm{mg}\right.$ ) in combination with chemotherapy (nab-paclitaxel, $100 \mathrm{mg} / \mathrm{m}^{2} \mathrm{and}$ carboplatin, $5 \mathrm{mg} / \mathrm{ml}$ per $\mathrm{min}$ ) achieved MPR and pCR, respectively. Patients without disease progression after two cycles received a further two cycles prior to surgery [22]. In the Lung Cancer Mutation Consortium 3 Phase II study, perioperative treatment with atezolizumab monotherapy (1200 $\mathrm{mg}$ for two cycles) in patients with stage IB to IIIB NSCLC showed an MPR rate of 18\% (15/82); four patients achieved pCR [23]. The first results of PRINCEPS 


\begin{tabular}{|c|c|c|c|c|c|c|}
\hline Study name (year) & Phase & Stage & Neoadjuvant treatment & $\mathrm{MPR}^{\dagger} / \mathrm{pCR}$ & Patients (n) & Ref. \\
\hline Forde PM et al. (2018) & II & I-IIIA & Nivolumab ( $3 \mathrm{mg} / \mathrm{kg}$ for 2 cycles) & $45 \% \mathrm{MPR}$ & 21 & [20] \\
\hline Ready N et al. (2019) & II & I-IIIA & Pembrolizumab (200 mg for 2 cycles) & $\begin{array}{l}>7 \% \mathrm{pCR} \\
\text { MPR was achieved but } \\
\text { the number of patients (\%) was } \\
\text { not reported }\end{array}$ & 30 & [21] \\
\hline Shu CA et al. (2020) & II & IB-IIIA & $\begin{array}{l}\text { Atezolizumab (1200 } \mathrm{mg} \text { for } 2 \text { cycles) }+ \text { CTx } \\
\text { (nab-paclitaxel, } 100 \mathrm{mg} / \mathrm{m}^{2} \text { and carboplatin, } \\
5 \mathrm{mg} / \mathrm{ml} \text { per } \mathrm{min} \text { ) }\end{array}$ & $\begin{array}{l}57 \% \mathrm{MPR} \\
33 \% \mathrm{pCR}\end{array}$ & 30 & [22] \\
\hline LCMC3 (2019) & II & IB-IIIB & Atezolizumab (1200 mg for 2 cycles) & $\begin{array}{l}18 \% \text { MPR } \\
5 \% \text { pCR }\end{array}$ & 82 & [23] \\
\hline PRINCEPS (2020) & II & IA-IIIA & Atezolizumab (1200 mg for 1 cycle) & $13 \% \mathrm{MPR}$ & 30 & [24] \\
\hline NADIM (2020) & II & IIIA & $\begin{array}{l}\text { Nivolumab ( } 360 \mathrm{mg} \text { for } 3 \text { cycles) }+ \text { CTx } \\
\text { (paclitaxel } 200 \mathrm{mg} / \mathrm{m}^{2} \text { and carboplatin, } \\
6 \mathrm{mg} / \mathrm{ml} \text { per } \mathrm{min} \text { ) }\end{array}$ & $\begin{array}{l}83 \% \mathrm{MPR} \\
63 \% \mathrm{pCR}\end{array}$ & 41 & [25] \\
\hline NEOSTAR (2019) & II & I-IIIA & $\begin{array}{l}\text { Nivolumab ( } 3 \mathrm{mg} / \mathrm{kg} \text { for } 3 \text { cycles }) \pm \text { ipilimumab } \\
(1 \mathrm{mg} / \mathrm{kg} \text { for } 3 \text { cycles) }\end{array}$ & $\begin{array}{l}24 \% \mathrm{MPR} \\
15 \% \mathrm{pCR}\end{array}$ & 44 & [26] \\
\hline IONESCO $\ddagger(2020)$ & II & IB-IIIA & Durvalumab ( $750 \mathrm{mg}$ for 3 cycles) & $\begin{array}{l}\text { MPR was achieved but the } \\
\text { number of patients (\%) was not } \\
\text { reported }\end{array}$ & 46 & [27] \\
\hline
\end{tabular}

in 30 patients with resectable NSCLC (stage IA-IIIA, non-N2) reported an MPR rate of 13\% (4/30) with one cycle of $1200 \mathrm{mg}$ neoadjuvant atezolizumab. MPR correlated with high PD-L1 expression but not with overall response rate (ORR); neoadjuvant treatment was considered safe and did not impair surgery [24]. The combination of nivolumab (360 mg for three cycles) with chemotherapy (paclitaxel, $200 \mathrm{mg} / \mathrm{m}^{2}$ and carboplatin, $6 \mathrm{mg} / \mathrm{ml}$ per $\mathrm{min}$ ) as neoadjuvant treatment was investigated in the Phase II, single-arm NADIM trial in patients with stage IIIA resectable NSCLC; 83\% (34/41) of patients achieved MPR and 63\% (26/41) of patients achieved pCR [25]. Results of the Phase II NEOSTAR trial in 44 patients with resectable NSCLC (stage I-IIIA, single N2) showed an overall MPR rate of 24\% (10/41) with neoadjuvant nivolumab monotherapy $(3 \mathrm{mg} / \mathrm{kg}$ for three cycles during the 3-6 weeks before surgery; four patients) and in combination with ipilimumab $(1 \mathrm{mg} / \mathrm{kg}$ for three cycles during the 3-6 weeks before surgery; six patients); six patients had pCR (two with nivolumab and four with the combination treatment) [26]. A 90-day perioperative analysis of the NEOSTAR surgical outcomes reported that, overall, $22 \%(8 / 37)$ of operations were delayed beyond 42 days; of these, $14 \%(3 / 21)$ and $31 \%(5 / 16)$ of patients received nivolumab monotherapy and in combination with ipilimumab, respectively. Surgeons subjectively judged $40 \%$ (15/37) of operations to be more complex than usual [28]. A new phenomenon of apparent radiographic progression of lymph nodes without pathological evidence of tumor, defined as nodal immune flare, was identified in the NEOSTAR trial following neoadjuvant treatment with immune checkpoint inhibitors (ICIs). Overall, nodal immune flare occurred in 11\% (5/44) of patients; of these, 13\% (3/23) and 10\% (2/21) received nivolumab monotherapy or combination treatment, respectively [29]. Neoadjuvant treatment with three cycles of $750 \mathrm{mg}$ durvalumab was evaluated in IONESCO in 46 patients with resectable NSCLC (stage IB-IIIA, non-N2). The observed MPR was significantly associated with DFS. The trial was terminated early due to excessive postoperative mortality [27].

Close monitoring of the impact of neoadjuvant treatment in larger trials is needed to confirm safety and feasibility ICIs in this setting. Multiple ongoing Phase II and III clinical trials are further evaluating these aspects and the survival and pathologic response by neoadjuvant immune modulation (Table 2).

ICIs restore antitumor immune response by blocking inhibitory receptors such as PD-1 [30]. The PD-1 pathway is a key mediator of local immunosuppression in the TME; it can also modulate T cell priming against tumor antigens in secondary lymphoid tissues [11]. Another attractive pathway for modulating the TME is to neutralize pro-tumor inflammation (PTI) by inhibition of pro-inflammatory cytokines, for example, IL-1 $\beta$ [31]. IL-1 $\beta$ is an important pro-inflammatory factor for directing an immunosuppressive (anti-inflammatory) tumor environment that impedes immunosurveillance [32]. IL-1 $\beta$ can promote the infiltration of immunosuppressive cells into the TME 
Table 2. Selected ongoing trials of neoadjuvant immunotherapeutic agents in resectable non-small-cell lung cancer.

\begin{tabular}{|c|c|c|c|c|c|}
\hline Trial & Phase & Stage & Neoadjuvant treatment & $\begin{array}{l}\text { Selected primary end } \\
\text { point(s) }\end{array}$ & Target accrual \\
\hline $\begin{array}{l}\text { NCT02998528 } \\
\text { (CheckMate 816) }\end{array}$ & III & $|B-I| \mid A$ & Nivolumab + ipilimumab/CTx & $\begin{array}{l}\text { EFS } \\
\text { PCR }\end{array}$ & 350 \\
\hline $\begin{array}{l}\text { NCT03425643 } \\
\text { (KEYNOTE 671) }\end{array}$ & III & II-IIIB & Pembrolizumab + CTx & $\begin{array}{l}\text { EFS } \\
\text { OS }\end{array}$ & 786 \\
\hline $\begin{array}{l}\text { NCT03456063 } \\
\text { (IMpower 030) }\end{array}$ & III & $\|-\| I B$ & Atezolizumab $+\mathrm{CTx}$ & $\begin{array}{l}\text { MPR } \\
\text { EFS }\end{array}$ & 374 \\
\hline $\begin{array}{l}\text { NCT04025879 } \\
\text { (CHECKMATE 77T) }\end{array}$ & III & $\|-\| I B$ & CTx + nivolumab & EFS & 452 \\
\hline $\begin{array}{l}\text { NCT03968419 } \\
\text { (CANOPY-N) }\end{array}$ & $\|$ & IB-IIIA & $\begin{array}{l}\text { Canakinumab } \pm \\
\text { pembrolizumab }\end{array}$ & MPR & 110 \\
\hline
\end{tabular}

CTx: Chemotherapy; EFS: Event-free survival; MPR: Major pathological response; NSCLC: Non-small-cell lung cancer; OS: Overall survival; pCR: Pathological complete response.

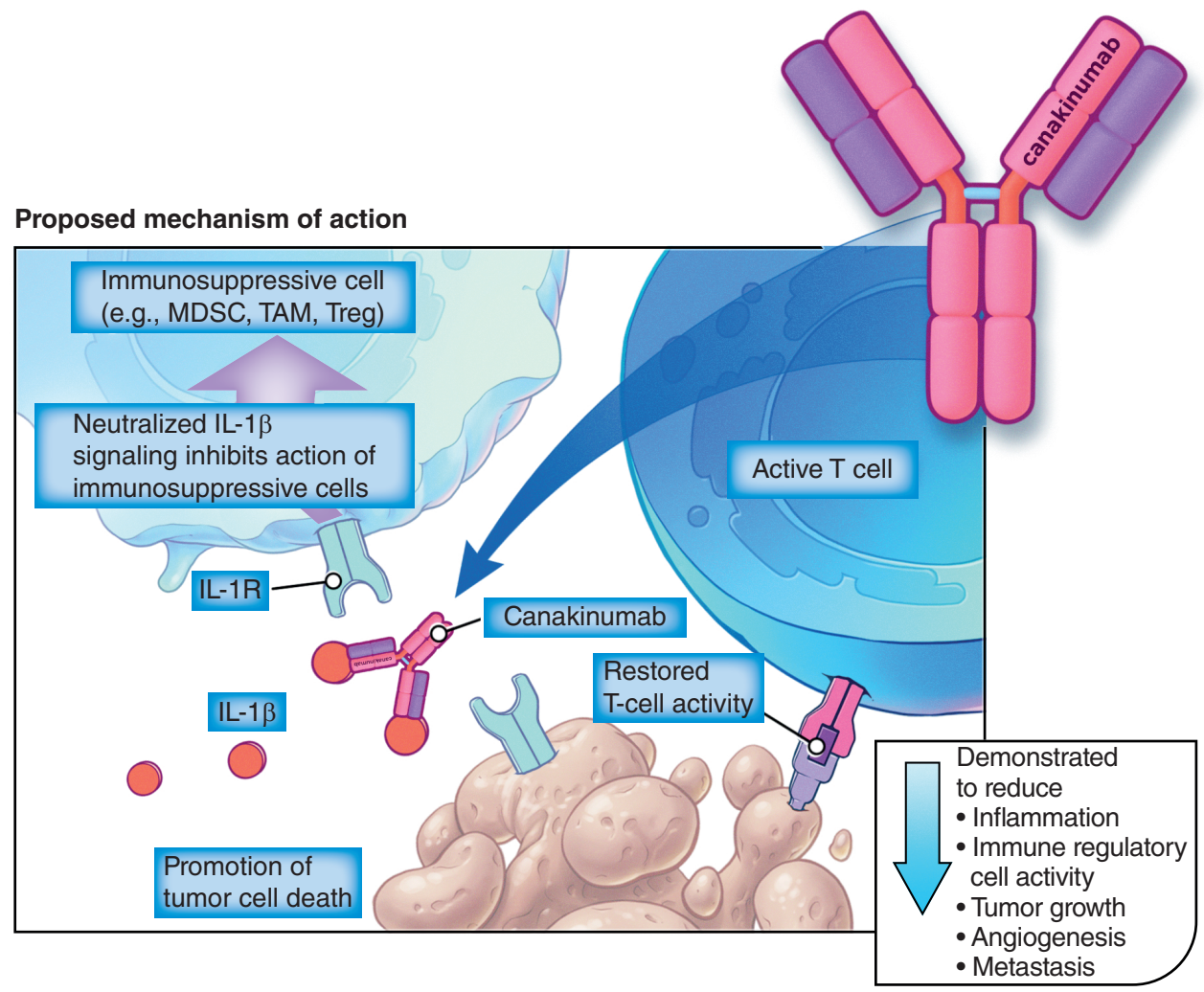

Figure 1. Canakinumab mechanism of action.

MDSC: Myeloid-derived suppressor cell; TAM: Tumor-associated macrophage; Treg: Regulatory T cell.

Reproduced with permission from (c) Novartis.

- including myeloid-derived suppressor cells, regulatory $\mathrm{T}$ cells and tumor-associated macrophages - and IL-1 $\beta$ plays a critical role in angiogenesis [33,34]. The synergistic effect of targeting different immune inhibitory pathways could overcome resistance and improve outcomes. In particular, the combination of IL-1 $\beta$ and PD- 1 inhibition has a synergistic effect by modulating TME cell populations in favor of the anti-tumorigenic phenotype [35,36].

Canakinumab is a human immunoglobulin (Ig) GK monoclonal antibody with high affinity and specificity for IL-1 $\beta$ [37,38]. Canakinumab neutralizes IL-1 $\beta$ activity by blocking its interaction with the IL-1 receptor [38-40]. By neutralizing IL-1 $\beta$, canakinumab inhibits PTI to enhance anti-tumor immune responses, reduce tumor cell proliferation, survival and invasiveness, and impair angiogenesis (Figure 1) [34,41]. Canakinumab is approved for 
the treatment of periodic fever syndromes and systemic juvenile idiopathic arthritis [38]. Canakinumab is also being studied as a single agent or in combination with other agents in patients across various therapeutic areas, including NSCLC, cytokine release syndrome in COVID-19-induced pneumonia, renal cell carcinoma, colorectal cancer, triple-negative breast cancer and cardiovascular (CV) disease [37].

The investigation of IL-1 $\beta$ inhibition with canakinumab as a therapeutic target in NSCLC was prompted by the results of the Canakinumab Anti-Inflammatory Thrombosis Outcome Study (CANTOS) trial. In this Phase III study, canakinumab was evaluated in the secondary prevention of $\mathrm{CV}$ events in post-myocardial infarction patients with CRP $\geq 2 \mathrm{mg} / \mathrm{l}$. The primary efficacy end point was non-fatal myocardial infarction, nonfatal stroke, or CV death. The primary end point was met by the canakinumab $150 \mathrm{mg}$ group, in which a risk reduction of $15 \%$ (hazard ratio [HR]: 0.85; 95\% CI: 0.74-0.98; adjusted $\mathrm{p}=0.021$ ) was demonstrated [42,43]. Study participants were followed up prospectively for incident adverse events (AEs), including investigator-reported malignancies. The risk of developing a malignancy was similar between the combined canakinumab arms and the placebo arm (HR: 0.93; 95\% CI: 0.79-1.09). Among the 685 participants who developed a malignancy, 129 were diagnosed with primary lung cancer. Compared with placebo, lung cancer occurrence was lower in the canakinumab arms $[37,43,44]$.

The CANTOS study demonstrated that blocking IL-1 $\beta$-driven inflammation in CV disease could prevent recurrent $\mathrm{CV}$ events in patients with persistent pro-inflammatory responses. Although primarily designed to evaluate CV events, CANTOS provided the first evidence of reduced incidence of and mortality from NSCLC using IL-1 $\beta$ inhibition, a finding consistent with experimental data on the role of IL-1 $\beta$-driven inflammation in cancer progression [35,36]. In a preplanned, exploratory post-hoc analysis, canakinumab treatment significantly reduced the incidence of lung cancer (HR: 0.33; 95\% CI: 0.18-0.59; p < 0.0001) and lung cancer mortality (HR: 0.23 ; 95\% CI: $0.10-0.54 ; \mathrm{p}=0.0002$ ) in the canakinumab $300 \mathrm{mg}$ group compared with placebo in a dose-dependent manner. Additionally, patients who were subsequently diagnosed with lung cancer were older $(\mathrm{p}<0.0001)$, more likely to be current smokers $(\mathrm{p}<0.0001)$ and had baseline concentrations of high-sensitivity CRP (hs-CRP; median 6.0 vs $4.2 \mathrm{mg} / \mathrm{l} ; \mathrm{p}<0.0001$ ) and IL-6 (3.2 vs $2.6 \mathrm{ng} / \mathrm{l} ; \mathrm{p}<0.0001$ ) significantly higher compared with those who were not diagnosed with cancer. Canakinumab treatment also resulted in a dose-dependent decrease in hs-CRP of 26-41\% and an IL-6 decrease of $25-43 \%$ [43].

These findings were supported by previous studies showing a negative association between elevated IL-1 $\beta$ levels and NSCLC progression and survival [45]. The role of IL-1 $\beta$ in tumorigenesis together with the hypothesis that canakinumab reduced the rate of progression, invasiveness and metastatic spread of subclinical tumors, provided the rationale to investigate IL-1 $\beta$ as a therapeutic target in different NSCLC settings in ongoing Phase III studies [46]. Multiple mechanisms could mediate the antineoplastic effects of IL-1 $\beta$ blockade at different stages of the disease, thus providing a new therapeutic target to be explored that may address unmet needs of patients with NSCLC. Conversely, the IL-1 $\beta$ pathway has redundancy with other cytokines and PTI may be driven by alternative pathways in some patient populations [47].

Considering that a systemic and locoregional inflammatory response is induced in the host from the earliest stages of cancer development, assessing the potential of IL-1 $\beta$ blockade in the neoadjuvant setting when the tumor mass and locoregional lymph nodes are intact is a possibility [48]. In a recent, retrospective bi-center study to assess the association between baseline CRP levels and anti-PD-1/PD-L1 treatment in patients with advanced NSCLC (discovery cohort, $\mathrm{n}=90$; validation cohort, $\mathrm{n}=101$ ), pretreatment CRP level was confirmed to predict progressionfree survival and OS [49]. Results of a retrospective analysis of 87 patients with advanced NSCLC receiving anti-PD-1 treatment demonstrated a significant association of baseline CRP with OS after immunotherapy [50]. In a study of 296 patients with NSCLC, elevated CRP at baseline was predictive of poorer progression-free survival with nivolumab treatment in patients with good Eastern Cooperative Oncology Group (ECOG) performance status (PS) of 0 or 1 [51]. In a study of 508 patients with advanced NSCLC, elevated CRP was predictive of poorer survival with nivolumab and was associated with PD-L1 expression [52].

The correlations observed between CRP levels (as a surrogate for IL-1 $\beta$ ) and PD-L1 expression, coupled with evidence that CRP level is negatively associated with survival in patients with advanced NSCLC, provided a rationale to evaluate whether neoadjuvant canakinumab could enhance the activity of ICIs. This hypothesis was supported by preclinical data, which showed that canakinumab in combination with pembrolizumab reduced tumor growth compared with either canakinumab or pembrolizumab monotherapy in a humanized NSCLC mouse model [53].

Canakinumab in combination with checkpoint inhibitors is a key clinical research area in both early and later-line settings due to the complex interplay between immunity and inflammation across all stages of tumor development [37]. In the neoadjuvant setting, a response to treatment could be observed as early as 4-6 weeks; 
previous studies reported early MPRs with ICIs at 4 weeks and a sustained reduction in CRP with canakinumab at approximately 7 days [20]. Previous studies reported improved survival with neoadjuvant chemotherapy in patients who achieved an MPR rate of approximately $20 \%$ at the time of surgery [19]. A $10 \%$ absolute improvement to a $30 \%$ MPR rate and a $25 \%$ absolute improvement to a $45 \%$ MPR rate will be considered of clinical benefit with canakinumab monotherapy and in combination with pembrolizumab, respectively.

\section{CANOPY-N trial}

Here, we present the design of the open-label, Phase II, randomized trial CANOPY-N (ClinicalTrials.gov: NCT03968419), evaluating the effect of canakinumab or pembrolizumab as monotherapy or in combination as neoadjuvant treatment in patients with resectable NSCLC [54].

\section{Objectives}

The primary objective of this study is to evaluate the effect of canakinumab versus canakinumab in combination with pembrolizumab as neoadjuvant (presurgery) immunotherapy for patients with early-stage NSCLC. This will be based on MPR rate ( $\leq 10 \%$ of residual viable tumor cells) at the time of surgery by central review [19]. Secondary objectives include: ORR based on local investigator assessment per Response Evaluation Criteria In Solid Tumors (RECIST) 1.1; MPR based on the levels of biomarkers, for example, PD-L1, CD8, hs-CRP, hs-IL-6; MPR by local review; surgical feasibility rate; safety; pharmacokinetics $(\mathrm{PK})$ and prevalence at baseline and incidence on-treatment of anti-drug antibodies.

\section{Key eligibility criteria}

Eligible patients aged $\geq 18$ years with histologically confirmed stage IB-IIIA NSCLC per American Joint Committee on Cancer 8th edition, deemed suitable for primary resection by the treating surgeon (except N2 and T4 tumors) are being enrolled. Patients must have an ECOG PS of 0 or 1, adequate organ function per laboratory assessments and be eligible for surgery.

A mandatory newly-obtained tissue biopsy from the primary site is required for study enrollment. An archival biopsy is also acceptable if obtained up to 5 months before the first day of study treatment and if the patient did not receive anti-neoplastic systemic therapies between the biopsy collection date and the beginning of study treatment. Other eligibility criteria are listed in Box 1.

\section{Study design}

CANOPY-N is a Phase II, randomized, open-label study (Figure 2). Approximately 110 patients will be randomized 2:2:1 to receive canakinumab $200 \mathrm{mg}$ subcutaneously once every 3 weeks (Q3W) monotherapy or in combination with pembrolizumab $200 \mathrm{mg}$ intravenously (iv.) Q3W or pembrolizumab monotherapy (200 mg iv. Q3W). Participants will be treated for up to 6 weeks (two cycles) and then submitted to surgery unless progression, unacceptable toxicity or discontinuation from the study for any other reason occurs. Live vaccines, biologic drugs targeting the immune system or immune-modulating agents in doses with systemic effects are prohibited for the duration of the study and $\geq 130$ days after canakinumab discontinuation due to the potential increase in immunosuppressant-related concomitant conditions.

The primary analysis will be performed after all patients have had surgical resection or have discontinued study treatment. The primary end point is the MPR rate at the time of surgery based on central review with canakinumab treatment with and without pembrolizumab. Secondary end points include ORR, MPR rate per local review in each treatment arm, MPR rate per central review in the pembrolizumab arm, surgical feasibility rate (percentage of patients who undergo surgery following study treatment), anti-drug antibody (ADA) prevalence at baseline, ADA incidence on-treatment, safety and PK. CANOPY-N trial end points are summarized in Box 2.

\section{Evaluations}

Patients will undergo positron emission tomography and computed tomography (PET-CT) imaging assessments at screening and after two cycles of treatment/before surgery for the response evaluation according to RECIST criteria. The CT component of PET-CT may be used if of similar diagnostic quality to a contrast-enhanced CT performed without PET.

MPR rate will be assessed using the percentage of patients with $\leq 10 \%$ residual viable cancer cells in the resected specimen at the time of surgery. The response is evaluated locally by the investigator as per RECIST 1.1 at screening 
Box 1. Patient eligibility criteria in the CANOPY-N trial

Inclusion criteria

- Age $\geq 18$ years.

- Histologically confirmed NSCLC stage IB-IIIA (per AJCC 8th edition), deemed suitable for primary resection by treating surgeon, except for N2 and T4 tumors.

- Patient must be eligible for surgery and with a planned surgical resection in approximately 4-6 weeks (after the first dose of study treatment).

- Mandatory newly obtained tissue biopsy from primary site or archived within the last 5 months before the first day of study treatment for patients not treated with antineoplastic systemic therapies between biopsy collection date and beginning of study treatment.

- Laboratory measurements within the normal range:

- $\mathrm{ANC} \geq 1.5 \times 10^{9} /$;

- Platelets $\geq 100 \times 10^{9} /$;

- Hemoglobin >9 g/dl;

- Creatinine clearance $>45 \mathrm{ml} / \mathrm{min}$ (Cockcroft-Gault formula);

- Total bilirubin $\leq 1.5 \times$ ULN;

- AST $\leq 3 \times$ ULN;

- $A L T \leq 3 \times$ ULN;

- Serum amylase $\leq 2 \times$ ULN or pancreatic amylase $\leq 1.5 \times$ ULN.

- Patients must have adequate cardiovascular and respiratory function to be submitted to surgical procedure as assessed per local clinical practice.

- ECOG PS of 0 or 1 .

Exclusion criteria

- Patients with unresectable or metastatic disease or brain metastasis.

- Increased risk of serious infusion reaction as assessed by the investigator.

- Presence or history of a malignant disease that has been diagnosed and/or required therapy within the past 3 years (excluding completely resected basal cell or squamous cell skin cancer or carcinoma in situ).

- Patients treated with any systemic therapy in the past 3 years before screening.

- Patients with active autoimmune disease that has required systemic treatment in the past 2 years before randomization (i.e., with use of disease modifying agents, corticosteroids or immunosuppressive drugs). Control of the disorder with replacement therapy (e.g., thyroxine, insulin or physiologic corticosteroid replacement therapy for adrenal or pituitary insufficiency) is permitted.

- Uncontrolled diabetes as defined per the investigator.

- History of (noninfectious) pneumonitis that required steroids or current pneumonitis.

- Clinically significant heart disease and/or a cardiac event within 6 months.

- Major surgery within 4 weeks before randomization.

- Immunocompromised subjects.

- Live vaccination within 3 months before randomization.

- Women of child-bearing potential unless using highly effective contraception during the study and for 4 months after stopping study treatment; pregnant, lactating or women who plan to become pregnant during the study.

- Subject has any other concurrent severe and/or uncontrolled medical condition that would, in the investigator's judgment, cause unacceptable safety risks, contraindicate subject participation in the clinical study, or compromise compliance with the protocol (e.g., chronic pancreatitis, uncontrolled diabetes mellitus).

AJCC: American Joint Committee on Cancer; ANC: Absolute neutrophil count; ECOG: Eastern Cooperative Oncology Group; NSCLC: Non-small-cell lung cancer; PS: Performance status; ULN: Upper limit of normal.

and before surgery by CT scan. Safety will be assessed throughout and at every visit through physical examination, ECOG PS, vital signs, body weight, laboratory analysis and electrocardiogram.

Blood samples will be collected at various time points to evaluate the exposure and immunogenicity of canakinumab monotherapy and in combination with pembrolizumab in patients with NSCLC. The relationship between canakinumab PK parameters, for example, coefficient of variation and suppression of inflammatory blood markers, such as hs-CRP and hs-IL-6, will be assessed. The association between the levels of biomarkers, including the immune tumor markers PD-L1 and CD8, and the percentage of residual tumor cells at resection will be evaluated.

The frequency of treatment-emergent AEs will be assessed pretreatment from the day the patient provides informed consent to the day before the first treatment. Treatment-emergent AEs will also be assessed on-treatment 


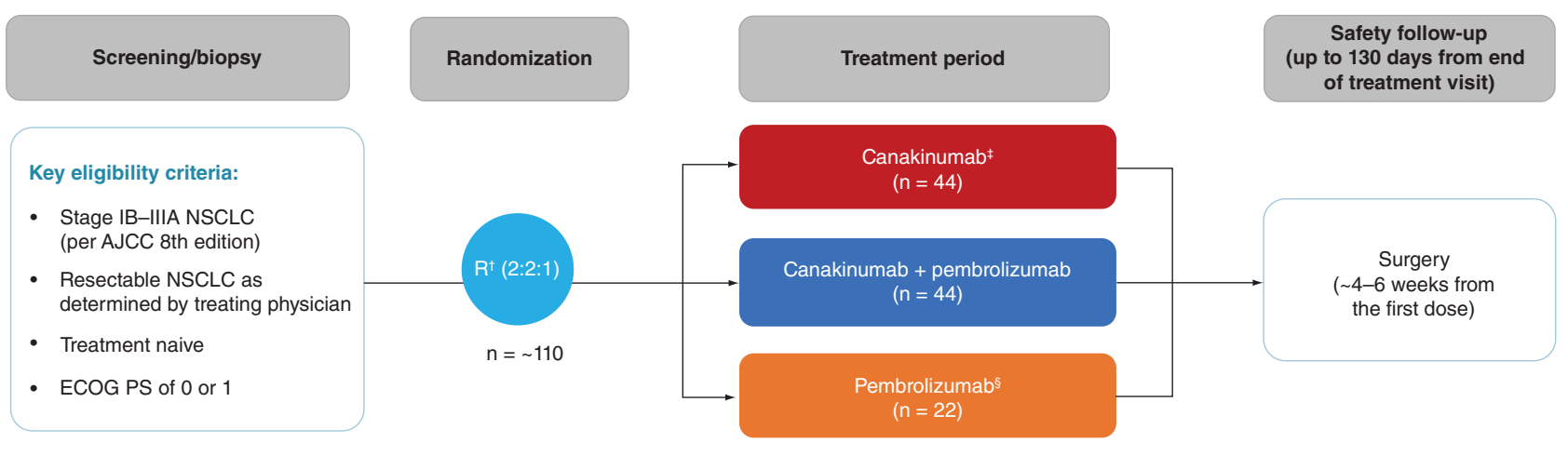

Primary end point: MPR rate at the time of surgery per central review in the canakinumab arm and the canakinumab + pembrolizumab arm

Key secondary end points: ORR; MPR rate per local review in each treatment arm; MPR rate per central review in the pembrolizumab arm; surgical feasibility rate (percentage of patients who undergo surgery following study treatment); ADA prevalence at baseline and ADA incidence on-treatment; safety; PK

Figure 2. CANOPY-N study design.

${ }^{\dagger}$ Randomization stratification: histology (squamous vs nonsquamous).

¥Canakinumab: two doses $200 \mathrm{mg}$ sc Q3W.

§embrolizumab: two doses 200 mg iv. Q3W.

ADA: Anti-drug antibody; AJCC: American Joint Committee on Cancer; ECOG: Eastern Cooperative Oncology Group; iv.: Intravenously; MPR: Major pathological response; NSCLC: Non-small-cell lung cancer; ORR: Overall response rate; PK: Pharmacokinetics; PS: Performance status; Q3W: Once every 3 weeks; sc: Subcutaneously.

\section{Box 2. CANOPY-N study end points}

\section{Primary end points}

- $\mathrm{MPR}^{\dagger}$ rate based on central review in the canakinumab monotherapy and combination with pembrolizumab arms.

Secondary end points

- ADA prevalence at baseline and ADA incidence on-treatment.

- Overall response rate based on local investigator assessment per RECIST 1.1.

- Concentrations of canakinumab, pembrolizumab.

- Surgical feasibility rate.

- MPR based on central review in the pembrolizumab arm or local review in all treatment arms.

- Difference in MPR rate between treatment arms based on central review.

- Type, frequency and severity of AEs.

- MPR based on the level of biomarkers (PD-L1, CD8, hs-CRP, hs-IL-6) assessed at baseline and on treatment.

Exploratory end points

- Association between canakinumab PK and suppression of inflammatory markers, such as hs-CRP and hs-IL-6; association between canakinumab PK and safety such as AEs and lab abnormalities; association between canakinumab PK and MPR.

- Association between the levels of biomarkers and percentage of residual tumor cells at resection.

- MPR based on the levels of blood biomarkers (e.g., cytokines, T-cell clonality, mutation status) and imaging PET scan.

- MPR based on the immune status of the tumor and the tumor microenvironment (e.g., WES including TMB, RNA sequencing) in samples collected at baseline and at time of surgery.

- Surgery-related safety end points.

$\dagger$ MPR was defined as $\leq 10 \%$ of residual viable tumor cells.

ADA: Anti-drug antibodies; AE: Adverse event; CD8: Cluster of differentiation 8; hs-CRP: High-sensitivity C-reactive protein; hs-IL-6:

High-sensitivity IL-6; MPR: Major pathological response; PET: Positron emission tomography; PK: Pharmacokinetics; RECIST: Response

Evaluation Criteria In Solid Tumors; TCR: T-cell receptor; TMB: Tumor mutational burden; WES: Whole-exome sequencing. 
from the day of the first dose to 130 days after last dose of study medication and post-treatment from day 131 after the last dose of any study treatment.

\section{Statistical analyses methods}

A total of 110 patients will be randomized 2:2:1 to one of the treatment arms. The MPR rate primary end point and its corresponding two-sided 95\% CI will be measured in the full analysis set comprising all patients who were randomly assigned. ORR is defined as the percentage of patients with a best overall response of complete response or partial response at the end of treatment visit prior to the surgery, as assessed using RECIST 1.1 per local review. ORR and two-sided exact binomial 95\% CI will be presented by treatment group. Patients with a best overall response of 'unknown' by RECIST 1.1 will be considered as nonresponders when estimating ORR. The incidence of treatment-emergent AEs will be summarized by system organ class and/or preferred term, severity based on Common Terminology Criteria for Adverse Events (CTCAE) 5.0 and relationship to study treatment by investigator. Laboratory measurements will be assigned grades programmatically per CTCAE 5.0 where applicable or categorized as low/normal/high based on laboratory normal ranges.

\section{Discussion}

A considerable body of evidence indicates that IL-1 $\beta$ plays a key role in driving cancer progression and immune suppression through multiple mechanisms [55]. Canakinumab is the first IL-1 $\beta$ inhibitor to be investigated for the treatment of lung cancer. IL-1 $\beta$ plays key roles to drive PTI-enabled tumor progression and suppress antitumor responses. IL-1 $\beta$ activates NF- $\mathrm{KB}$ and STAT3, leading to increased expression of pro-inflammatory molecules, such as IL-6, IL-8, CCL2 and COX-2, all of which have reported roles in tumorigenesis [41]. IL-1 $\beta$ promotes metastasis by mediating epithelial-to-mesenchymal transition and stimulating the expression of matrix metalloproteases and other molecules with roles in matrix degradation, cell motility and adhesion [56]. IL-1 $\beta$ promotes tumor angiogenesis by mediating endothelial cell migration and expression of VEGF and its receptors $[34,41,57]$.

By neutralizing IL-1 $\beta$, canakinumab inhibits PTI, thereby enhancing anti-tumor immune responses; reducing tumor cell proliferation, survival and invasiveness, and impairing angiogenesis [34,41]. Canakinumab being used as a single agent in the CANTOS trial has led to a reduction in CRP from baseline [43]. Several studies reported improved survival in patients with advanced NSCLC treated with anti-PD-L1 inhibitors who experienced a reduction in CRP level [49].

The CANOPY-N trial is the first Phase II trial of an IL-1 $\beta$ inhibitor in the neoadjuvant setting in patients with NSCLC. Enrollment began in November 2019, and the estimated completion date of the study primary analysis is September 2021. Results from this trial will determine whether canakinumab monotherapy or canakinumab in combination with an ICI, can offer a treatment alternative to be further explored as a neoadjuvant therapy with increased efficacy and reduced toxicities.

One of the areas of major debate in the NSCLC neoadjuvant setting is the role of MPR as a primary end point. MPR has been used as a surrogate efficacy end point in several neoadjuvant immunotherapy trials. However, the unification of MPR evaluation standards can be problematic and the assessment of the degree of residual cancer is only semi-quantitative $[17,19]$. Challenges include variability in the pathologists' recordings of MPR, tumor heterogeneity, differential implications of this metric between the histological subtypes (i.e., adenocarcinoma or squamous cell carcinoma), and the possibility that the $10 \%$ cut-off may not be suitable for immunotherapies. The study selected MPR as the primary end point to allow for a more efficient and objective evaluation of canakinumab. This follows the most recent international recommendations for pathologic assessment, which provide detailed guidance on how to process lung cancer resection specimens after neoadjuvant therapy [19]. MPR provides the possibility of an earlier assessment of the direct anti-tumor effect of treatment, quantifiable right after surgery, in contrast to DFS and OS, which are long-term end points. This is a particularly important consideration for small Phase II trials, where many patients lost to follow-up can impact on the significance of the study results. Additionally, because more research is needed to identify optimal biomarkers to best individualize neoadjuvant treatment approaches, the trial is also assessing MPR based on the level of key blood and tissue biomarkers (e.g., PD-L1, CD8, hs-CRP and hs-IL-6) as a secondary end point.

Because concerns remain regarding the potential of neoadjuvant ICIs for complications and delays at the time of curative surgery, large ongoing trials aim to confirm the safety of their preoperative use. In other settings, adverse reactions from immunotherapy may occur months to years after the last dose. This finding highlights the need for patient monitoring or new immuno-oncology targets, or combination treatments that minimize adverse 
side effects and toxicities in the long term, and also highlights the need for an increased understanding of the immunomodulators mechanisms of action [58]. The safety and tolerability of canakinumab have been demonstrated in multiple indications in clinical trials and by real-world data $[59,60]$. The most frequently reported AEs in all indications are infections, predominantly of the upper respiratory tract, including serious infections, which have been reported more frequently with canakinumab than with placebo or comparators. Inhibiting IL-1 $\beta$ receptor signaling may alter immunologic responsiveness in unanticipated ways and vigilant monitoring is recommended in case of conditions with immune suppression or dysregulation, such as malignancies.

CANOPY-N is part of the CANOPY clinical trial program designed to evaluate canakinumab efficacy and safety in different NSCLC settings and/or combination treatments [46]. It consists of the Phase II CANOPY$\mathrm{N}$ trial in the neoadjuvant setting, which aims to manage distant micro-metastases following primary tumor resection, and three Phase III randomized, double-blind, placebo-controlled trials in the adjuvant (CANOPYA, NCT03447769) [61], first-line advanced (CANOPY-1, NCT03631199) [62] and second-/third-line treatment settings (postimmunotherapy and platinum-based chemotherapy, either sequentially or combined) (CANOPY-2, NCT03626545).

The CANOPY program aims to identify the optimal settings and patient populations for maximal benefit of IL-1 $\beta$ inhibition. In neoadjuvant NSCLC, canakinumab may have clinical utility either used as a monotherapy or in synergy with anti-PD-1 therapy.

\section{Conclusion}

The CANOPY-N trial will evaluate whether the addition of pembrolizumab enhances the activity of canakinumab. The trial aims to better understand canakinumab's mechanism of action in an oncological setting and explore the clinical utility of potential predictive biomarkers. To understand the molecular mechanisms through which IL-1 $\beta$ inhibition can prevent or reduce tumor growth and whether it synergizes with anti-PD-L1 treatment, the benefits of each treatment regimen as monotherapy or in combination will be assessed. Considering the overall safety profile of canakinumab and the MPR rates with neoadjuvant pembrolizumab treatment in NSCLC, it is hoped that canakinumab in combination with pembrolizumab can inform clinical development of new neoadjuvant NSCLC treatments to address an unmet medical need.

Author contributions

All authors met the criteria for authorship set forth by the International Committee of Medical Journal Editors, and were involved in conception, preparation and approval of the manuscript.

\section{Acknowledgments}

The authors would like to thank the patients and their families, investigators, co-investigators, and the study teams at each of the participating centers.

\section{Financial \& competing interests disclosure}

P Garrido reports personal fees from Roche, Merck Sharp \& Dohme Corp, Bristol Myers Squibb, Boehringer-Ingelheim, Pfizer, Abbvie, Novartis, Eli Lilly, AstraZeneca, Janssen Pharmaceuticals, Blueprint Medicines, Takeda Pharmaceutical Company, Gilead Sciences, Rovi; JM Lee reports personal fees from AstraZeneca, Bristol Myers Squibb, Genentech and Novartis, participation in Executive/Steering Committee for Genentech, Novartis and Research Support from Merck \& Co.; M Tsuboi received personal fees from Johnson \& Johnson, AstraZeneca, Eli Lilly, Chugai Pharmaceutical, Merck Sharp \& Dohme Corp, Bristol Myers Squibb, Teijin Pharma, Taiho Pharma, Medtronic, Ono Pharmaceutical, Novartis, grants from Boehringer-Ingelheim, Merck Sharp \& Dohme Corp, AstraZeneca, Ono Pharmaceutical, Bristol Myers Squibb, Eli Lilly; TSK Mok reports personal fees from Abbvie, Inc., MD Health (Brazil), Medscape/WebMD, MoreHealth, PeerVoice, Physicians' Education Resource, Permanyer SL, PrIME Oncology, Research to Practice, Touch Medical Media, personal fees and other from ACEA Pharma, Alpha Biopharma Co. Ltd., Amgen, Amoy Diagnostics Co. Ltd., BeiGene, Boehringer Ingelheim, Blueprint Medicines Corporation, CStone Pharmaceuticals, Daiichi Sankyo, Eisai, Merck Serono, Merck Sharp \& Dohme, Mirati Therapeutics Inc., OrigiMed, Puma Technology Inc, Roche, Sanofi-Aventis R\&D, Takeda, Yuhan Corporation, grants, personal fees and other from AstraZeneca, Bristol-Myers Squibb, Fishawack Facilitate Ltd., geneDecode, Gritstone Oncology Inc, Guardant Health, Hengrui Therapeutics, Ignyta Inc., QVIA, InMed Medical Communication, Incyte Corporation, Janssen, Eli Lilly, Loxo-Oncology, Lunit, Inc., Novartis, Pfizer Inc; grants from Clovis Oncology, SFJ Pharmaceuticals and XCovery, other financial support from Virtus Medical Group, AstraZeneca PLC, Hutchison Chi-Med, Sanomics Ltd. C Blin is a Novartis Pharma AG employee. T Dong and VR Outmezguine are Novartis Pharmaceutical Corp employees. VQ Passos is an employee 
of and holds stocks in Novartis Pharmaceutical Corp. JL Pujol, ES Kim, AG Rueda, A Benito, N Moreno and L Gorospe have nothing to disclose. The authors have no other relevant affiliations or financial involvement with any organization or entity with a financial interest in or financial conflict with the subject matter or materials discussed in the manuscript apart from those disclosed.

The authors thank Oana Coban, PhD, of Chameleon Communications for providing medical writing, which was funded by Novartis Pharmaceuticals in accordance with Good Publication Practice (GPP3) guidelines (http://www.ismpp.org/gpp3). Financial support for medical editorial assistance was provided by Novartis Pharmaceuticals Corporation.

\section{Ethical conduct of research}

This study was conducted in accordance with the principles of the Declaration of Helsinki and the Good Clinical Practice guideline of the International Conference on Harmonization. The study protocol and all amendments were reviewed by the independent ethics committee or institutional review board at each center and all patients provided written informed consent. The study protocol and statistical analysis plan are available with the full text of this article. The study was sponsored by Novartis Pharmaceuticals Corporation and was designed in conjunction with an independent steering committee. The sponsor conducted all statistical analyses. All authors agreed to submit the manuscript for publication and vouch for its accuracy. The manuscript was developed with medical writing support funded by the sponsor.

\section{Data sharing statement}

Novartis is committed to sharing with qualified external researchers, access to patient-level data and supporting clinical documents from eligible studies. These requests are reviewed and approved by an independent review panel on the basis of scientific merit. All data provided is anonymized to respect the privacy of patients who have participated in the trial in line with applicable laws and regulations. This trial data availability is according to the criteria and process described on http://www.clinicalstudydatarequest.com.

\section{Open access}

This work is licensed under the Attribution-NonCommercial-NoDerivatives 4.0 Unported License. To view a copy of this license, visit http://creativecommons.org/licenses/by-nc-nd/4.0/

\section{Executive summary}

\section{Background}

- Patients with early-stage non-small-cell lung cancer (NSCLC) who have undergone surgical resection with curative intent frequently relapse due to distant metastasis, highlighting the need for new systemic treatments.

- Neoadjuvant chemotherapy demonstrated modest survival improvements in this group of patients.

- To decrease NSCLC recurrence rates, there is a need to test new therapeutic targets and combination treatments in this setting.

Canakinumab

- Canakinumab is a human IgGk monoclonal antibody with high affinity and specificity for IL-1 $\beta$, and is approved for the treatment of periodic fever syndromes and systemic juvenile idiopathic arthritis.

- In a preplanned, exploratory analysis of the Canakinumab Anti-Inflammatory Thrombosis Outcome Study (CANTOS), canakinumab treatment significantly reduced the incidence of lung cancer and lung cancer mortality in a dose-dependent manner (hazard ratio: $0.33 ; 95 \% \mathrm{Cl}: 0.18-0.59 ; \mathrm{p}<0.0001$ and hazard ratio: $0.23 ; 95 \% \mathrm{Cl}$ : $0.10-0.54 ; p=0.0002$, respectively).

- Canakinumab inhibits the IL-1 $\beta$ driven pro-tumor inflammation and has the potential to limit/perturb tumor progression and modulate the immunosuppressive tumor microenvironment to facilitate an anti-tumor immune response.

CANOPY-N trial

- CANOPY-N is the first Phase II trial to investigate neoadjuvant IL-1 $\beta$ inhibition with or without an immune checkpoint inhibitor in patients with resectable NSCLC.

- Eligible patients are $\geq 18$ years of age with histologically confirmed stage IB-IIIA NSCLC, per AJCC version 8, deemed suitable for primary resection by the treating surgeon (except N2 and T4 tumors).

- Patients are randomized 2:2:1 to receive canakinumab monotherapy or canakinumab in combination with pembrolizumab, or pembrolizumab monotherapy.

- Primary end point is major pathological response rate based on central review for canakinumab compared with canakinumab in combination with pembrolizumab.

- The results of this trial may demonstrate the potential value of IL-1 $\beta$ blockade alone or in combination with immune checkpoint inhibition to provide a new treatment option with improved curability and less toxicity for patients with resectable NSCLC. 


\section{References}

Papers of special note have been highlighted as: $\bullet$ of interest; $\bullet \bullet$ of considerable interest

1. International Agency for Research on Cancer. Cancer tomorrow. (2018). http://gco.iarc.fr/tomorrow/graphic-isotype

2. Bray F, Ferlay J, Soerjomataram I, Siegel RL, Torre LA, Jemal A. Global cancer statistics 2018: GLOBOCAN estimates of incidence and mortality worldwide for 36 cancers in 185 countries. CA Cancer J. Clin. 68(6), 394-424 (2018).

3. Molina JR, Yang P, Cassivi SD, Schild SE, Adjei AA. Non-small cell lung cancer: epidemiology, risk factors, treatment, and survivorship. Mayo Clin. Proc. 83(5), 584-594 (2008).

4. Howlader N, Noone AM, Krapcho M et al. SEER cancer statistics review (CSR) 1975-2017. (2020). https://seer.cancer.gov/csr/1975_2017/

5. Chiari R, Sidoni A, Metro G. Early stage resectable non-small cell lung cancer: is neoadjuvant immunotherapy the right way forward? J. Thorac. Dis. 10(Suppl. 33), S3890-S3894 (2018).

6. Blumenthal GM, Bunn PA Jr, Chaft JE et al. Current status and future perspectives on neoadjuvant therapy in lung cancer. J. Thorac. Oncol. 13(12), 1818-1831 (2018).

7. Wu CF, Fu JY, Yeh CJ et al. Recurrence risk factors analysis for stage I non-small cell lung cancer. Medicine (Baltimore) 94(32), e1337 (2015).

8. Kenmotsu H, Ohde Y, Wakuda $\mathrm{K}$ et al. Survival data for postoperative adjuvant chemotherapy comprising cisplatin plus vinorelbine after complete resection of non-small cell lung cancer. Cancer Chemother. Pharmacol. 80(3), 609-614 (2017).

9. NSCLC Meta-analysis Collaborative Group. Preoperative chemotherapy for non-small-cell lung cancer: a systematic review and meta-analysis of individual participant data. Lancet 383(9928), 1561-1571 (2014).

10. Song WA, Zhou NK, Wang W et al. Survival benefit of neoadjuvant chemotherapy in non-small cell lung cancer: an updated meta-analysis of 13 randomized control trials. J. Thorac. Oncol. 5(4), 510-516 (2010).

11. Topalian SL, Taube JM, Pardoll DM. Neoadjuvant checkpoint blockade for cancer immunotherapy. Science 367(6477), eaax0182 (2020).

12. Spigel D, de Marinis F, Giaccone G et al. IMpower110: Interim overall survival (OS) analysis of a Phase III study of atezolizumab (atezo) vs platinum-based chemotherapy (chemo) as first-line (1L) treatment (tx) in PD-L1-selected NSCLC. Ann Oncol. 30(Suppl. 5), v851-v934 (2019).

13. Reck M, Rodriguez-Abreu D, Robinson AG et al. Pembrolizumab versus chemotherapy for PD-L1-positive non-small-cell lung cancer. N. Engl. J. Med. 375(19), 1823-1833 (2016).

14. Antonia SJ, Villegas A, Daniel D et al. Overall survival with durvalumab after chemoradiotherapy in stage III NSCLC. N. Engl. J. Med. 379(24), 2342-2350 (2018).

15. Kris MG, Faivre-Finn C, Kordbacheh T et al. Making checkpoint inhibitors part of treatment of patients with locally advanced lung cancers: the time is now. Am. Soc. Clin. Oncol. Educ. Book 40, 1-12 (2020).

16. Melosky B, Juergens R, McLeod D et al. Immune checkpoint-inhibitors and chemoradiation in stage III unresectable non-small cell lung cancer. Lung Cancer 134, 259-267 (2019).

17. Ren S, Wang C, Shen J, Zhu C. Neoadjuvant immunotherapy with resectable non-small cell lung cancer: recent advances and future challenges. J. Thorac. Dis. 12(4), 1615-1620 (2020).

18. Pataer A, Kalhor N, Correa AM et al. Histopathologic response criteria predict survival of patients with resected lung cancer after neoadjuvant chemotherapy. J. Thorac. Oncol. 7(5), 825-832 (2012).

19. Travis WD, Dacic S, Wistuba I et al. IASLC multidisciplinary recommendations for pathologic assessment of lung cancer resection specimens after neoadjuvant therapy. J. Thorac. Oncol. 15(5), 709-740 (2020).

- Recommendations from the International Association for the Study of Lung Cancer on processing and evaluating lung cancer resection specimens to determine pathological response after neoadjuvant treatment.

20. Forde PM, Chaft JE, Smith KN et al. Neoadjuvant PD-1 blockade in resectable lung cancer. N. Engl. J. Med. 378(21), 1976-1986 (2018).

- A pilot study evaluating the safety and feasibility of preoperative programmed death-1 inhibition with nivolumab in patients with untreated, surgically resectable early non-small-cell lung cancer (NSCLC).

21. Ready N, Tong B, Clarke J et al. Neoadjuvant pembrolizumab in early stage non-small cell lung cancer (NSCLC): toxicity, efficacy, and surgical outcomes. J. Thorac. Oncol. 14(10), S745 (2019).

22. Shu CA, Gainor JF, Awad MM et al. Neoadjuvant atezolizumab and chemotherapy in patients with resectable non-small-cell lung cancer: an open-label, multicentre, single-arm, Phase II trial. Lancet Oncol. 21(6), 786-795 (2020).

23. Kwiatkowski DJ, Rusch VW, Chaft JE et al. Neoadjuvant atezolizumab in resectable non-small cell lung cancer (NSCLC): interim analysis and biomarker data from a multicenter study (LCMC3). J. Clin. Oncol. 37(Suppl. 15), 8503-8503 (2019).

24. Besse B, Adam J, Cozic N et al. SC neoadjuvant atezolizumab (A) for resectable non-small cell lung cancer (NSCLC): results from the Phase II PRINCEPS trial. Ann. Oncol. 31, S794-S795 (2020). 
25. Provencio M, Nadal E, Insa A et al. Neoadjuvant chemotherapy and nivolumab in resectable non-small-cell lung cancer (NADIM): an open-label, multicentre, single-arm, Phase II trial. Lancet Oncol. 21(11), 1413-1422 (2020).

26. Cascone T, William WN, Weissferdt A et al. Neoadjuvant nivolumab (N) or nivolumab plus ipilimumab (NI) for resectable non-small cell lung cancer (NSCLC): clinical and correlative results from the NEOSTAR study. J. Clin. Oncol. 37(Suppl. 15), 8504-8504 (2019).

27. Wislez M, Mazieres J, Lavole A et al. Neoadjuvant durvalumab in resectable non-small cell lung cancer (NSCLC): preliminary results from a multicenter study (IFCT-1601 IONESCO). Ann. Oncol. 31, S794 (2020).

28. Sepesi B, Cascone T, William W et al. Surgical outcomes following neoadjuvant nivolumab or nivolumab plus ipilimumab in non-small cell lung cancer - NEOSTAR study. J. Thorac. Oncol. 14, S241-S242 (2019).

29. Sepesi B, Godoy M, William W et al. Nodal immune flare (NIF) following neoadjuvant anti-PD-1 and anti-CTLA-4 therapy in non-small cell lung cancer. J. Thorac. Oncol. 14, S745 (2019).

-. A preliminary report of nodal immune flare, a distinct new phenomenon observed following neoadjuvant immune checkpoint inhibition, from the NEOSTAR Phase II trial.

30. Qin S, Xu L, Yi M, Yu S, Wu K, Luo S. Novel immune checkpoint targets: moving beyond PD-1 and CTLA-4. Mol. Cancer 18(1), 155 (2019).

31. Coussens LM, Zitvogel L, Palucka AK. Neutralizing tumor-promoting chronic inflammation: a magic bullet? Science 339(6117), 286-291 (2013).

32. Mantovani A, Barajon I, Garlanda C. IL-1 and IL-1 regulatory pathways in cancer progression and therapy. Immunol. Rev. 281(1), 57-61 (2018).

33. Fahey E, Doyle SL. IL-1 family cytokine regulation of vascular permeability and angiogenesis. Front. Immunol. 10(1426), 1426 (2019).

34. Carmi Y, Dotan S, Rider P et al. The role of IL-1 $\beta$ in the early tumor cell-induced angiogenic response. J. Immunol. 190(7), 3500-3509 (2013).

35. Kaplanov I, Carmi Y, Kornetsky R et al. Blocking IL-1 $\beta$ reverses the immunosuppression in mouse breast cancer and synergizes with anti-PD-1 for tumor abrogation. Proc. Natl Acad. Sci. USA 116(4), 1361-1369 (2019).

36. Baker KJ, Houston A, Brint E. IL-1 family members in cancer; two sides to every story. Front. Immunol. 10(1197), 1197 (2019).

37. Garon EB, Chih-Hsin Yang J, Dubinett SM. The role of interleukin 1b; in the pathogenesis of lung cancer. JTO Clin. Res. Rep. 1(1), 100001 (2020).

38. Novartis Pharmaceuticals Corporation. ILARIS (canakinumab) prescribing information. (2020). https://www.novartis.us/product/pi/pdf/ilaris.pdf

39. Alten R, Gram H, Joosten LA et al. The human anti-IL-1 beta monoclonal antibody ACZ885 is effective in joint inflammation models in mice and in a proof-of-concept study in patients with rheumatoid arthritis. Arthritis Res. Ther. 10(3), R67 (2008).

40. Rondeau JM, Ramage P, Zurini M, Gram H. The molecular mode of action and species specificity of canakinumab, a human monoclonal antibody neutralizing IL-1 $\beta$. MAbs 7(6), 1151-1160 (2015).

41. Voronov E, Apte RN. Targeting the tumor microenvironment by intervention in interleukin-1 biology. Curr. Pharm. Des. 23(32), 4893-4905 (2017).

42. Ridker PM, Thuren T, Zalewski A, Libby P. Interleukin-1 $\beta$ inhibition and the prevention of recurrent cardiovascular events: rationale and design of the Canakinumab Anti-inflammatory Thrombosis Outcomes Study (CANTOS). Am. Heart J. 162(4), 597-605 (2011).

43. Ridker PM, MacFadyen JG, Thuren T, Everett BM, Libby P, Glynn RJ. Effect of interleukin-1 $\beta$ inhibition with canakinumab on incident lung cancer in patients with atherosclerosis: exploratory results from a randomised, double-blind, placebo-controlled trial. Lancet 390(10105), 1833-1842 (2017).

-• First evidence from a randomized clinical trial that inhibition of IL-1 $\beta$ with canakinumab is associated with reduced incidence of fatal cancer, lung cancer and fatal lung cancer.

44. Ridker PM, Everett BM, Thuren T et al. Antiinflammatory therapy with canakinumab for atherosclerotic disease. N. Engl. J. Med. 377(12), 1119-1131 (2017).

45. Wu C, Xu B, Zhou Y et al. Correlation between serum IL-1 $\beta$ and miR-144-3p as well as their prognostic values in LUAD and LUSC patients. Oncotarget 7(52), 85876-85887 (2016).

46. Paz-Ares L, Garon EB, Ardizzoni A et al. CANOPY Phase III program: three studies evaluating canakinumab in patients with non-small cell lung cancer (NSCLC). Ann. Oncol. 30(Suppl. 5), v602 (2019).

- A description of the CANOPY program and individual study designs evaluating canakinumab in patients with NSCLC across different settings of unmet need.

47. Gram H. The long and winding road in pharmaceutical development of canakinumab from rare genetic autoinflammatory syndromes to myocardial infarction and cancer. Pharmacol. Res. 154, 104139 (2020).

48. Ghysen K, Vansteenkiste J. Immunotherapy in patients with early stage resectable nonsmall cell lung cancer. Curr. Opin. Oncol. 31(1), 13-17 (2019). 
49. Riedl JM, Barth DA, Brueckl WM et al. C-reactive protein (CRP) levels in immune checkpoint inhibitor response and progression in advanced non-small cell lung cancer: a bi-center study. Cancers (Basel) 12(8), 2319 (2020).

50. Naqash AR, Stroud CRG, Butt MU et al. Co-relation of overall survival with peripheral blood-based inflammatory biomarkers in advanced stage non-small cell lung cancer treated with anti-programmed cell death-1 therapy: results from a single institutional database. Acta Oncol. 57(6), 867-872 (2018).

51. Adachi $Y$, Tamiya A, Taniguchi $Y$ et al. Predictive factors for progression-free survival in non-small cell lung cancer patients receiving nivolumab based on performance status. Cancer Med. 9(4), 1383-1391 (2020).

52. Akamine T, Takada K, Toyokawa G et al. Association of preoperative serum CRP with PD-L1 expression in 508 patients with non-small cell lung cancer: a comprehensive analysis of systemic inflammatory markers. Surg. Oncol. 27(1), 88-94 (2018)

53. Jayaraman P, Millholland J, O Brien N et al. Targeting IL-1 $\beta$ pathway for cancer immunotherapy. Mol. Cancer Ther. 18(Suppl. 12), Abstract C103 (2019).

-• This preclinical study demonstrated the anti-tumor activity of canakinumab as a monotherapy or in combination with anti-PD1 agents in humanized mouse models of lung cancer.

54. Mok TSK, Garrido Lopez P, Kim ES et al. Randomized Phase II study of canakinumab (CAN) or pembrolizumab (PEM) as monotherapy or in combination as neoadjuvant therapy in patients (Pts) with surgically resected (stage IB-IIIA) non-small cell lung cancer (NSCLC): CANOPY-N. J. Clin. Oncol. 38(Suppl. 15), TPS9626-TPS9626 (2020).

55. Dinarello CA. Overview of the IL-1 family in innate inflammation and acquired immunity. Immunol. Rev. 281(1), 8-27 (2018).

56. Metro G, Banna GL, Signorelli D et al. Efficacy of pembrolizumab monotherapy in patients with or without brain metastases from advanced non-small cell lung cancer with a PD-L1 expression $\geq 50$. J. Immunother. 43(9), 299-306 (2020).

57. Voronov E, Carmi Y, Apte RN. The role IL-1 in tumor-mediated angiogenesis. Front. Physiol. 5, 114 (2014).

58. Marshall HT, Djamgoz MBA. Immuno-oncology: emerging targets and combination therapies. Front. Oncol. 8, 315 (2018).

59. Sota J, Vitale A, Insalaco A et al. Safety profile of the interleukin-1 inhibitors anakinra and canakinumab in real-life clinical practice: a nationwide multicenter retrospective observational study. Clin. Rheumatol. 37(8), 2233-2240 (2018).

60. Wulffraat NM. A safety evaluation of canakinumab for the treatment of systemic onset juvenile idiopathic arthritis. Expert Opin. Drug Saf. 14(12), 1961-1967 (2015).

61. Garon E. CANOPY-A: a Phase III, multicenter, randomized, double-blind, placebo-controlled trial evaluating canakinumab as adjuvant therapy in patients (pts) with completely resected non-small cell lung cancer (NSCLC). Am. Soc. Clin. Oncol. Virtual (2020) (Poster TPS9075).

62. Felip E, Castro G, Greystoke A et al. CANOPY-1: Phase III study of canakinumab/placebo + pembrolizumab + platinum-chemotherapy in untreated stage IIIB-IV NSCLC pts. J. Thorac. Oncol. 14(Suppl. 10), S645 (2019). 